\title{
Variables relevantes para el escepticismo profesional en la formación de contadores auditores
}

\author{
Joseline J. Sepúlveda-Araya*, Francisco A. Gálvez-Gamboa , Cristian Vilches-Meza y Hortensia Hinojosa-Moya \\ Facultad de Ciencias Sociales y Económicas, Universidad Católica del Maule, Av. San Miguel 3605, Talca, Chile. \\ (correo-e: joseline.sepulveda.araya@gmail.com, franziscogalvez@gmail.com, c.vilches.meza@gmail.com, \\ hinojosamoya.20@hotmail.com) \\ * Autor a quien debe ser dirigida la correspondencia.
}

Recibido Dic. 16, 2020; Aceptado Feb. 23, 2021; Versión final Abr. 12, 2021, Publicado Ago. 2021

\begin{abstract}
Resumen
El presente trabajo tuvo por objetivo analizar los determinantes del grado de escepticismo en los egresados de auditoría. Se aplicó una encuesta de percepción sobre el escepticismo profesional a 160 egresados de la carrera de auditoría de la Universidad Católica del Maule (Chile). Se analizaron las dimensiones de conocimiento, aplicación y formación sobre escepticismo profesional utilizando un modelo probabilístico del tipo probit ordenado. Los resultados indican que son relevantes de analizar el área de desempeño actual del egresado, tomando en consideración que desempeñarse en el área de auditoría es un moderador relevante del escepticismo en las dimensiones de concepto y aplicación. También es un determinante relevante la experiencia profesional, demostrando que modera las dimensiones de concepto y formación académica en escepticismo. Como conclusión, se puede indicar que la experiencia profesional es un moderador relevante del escepticismo profesional, por lo cual las actividades prácticas en auditoría son una estrategia que puede favorecer y potenciar la formación de los futuros egresados.
\end{abstract}

Palabras clave: escepticismo profesional; auditoría; formación universitaria; competencia; educación

\section{Variables relevant to professional skepticism in training accountants and auditors}

\begin{abstract}
The objective of this study was to analyze the determinants of the degree of skepticism among audit graduates. A perception survey on professional skepticism was applied to 160 audit graduates from the Universidad Católica del Maule (Chile). The dimensions of knowledge, application, and training on professional skepticism were analyzed using a probabilistic ordered-probit-type model. The results indicate that it is important to analyze the job field of each graduate since working in the area of auditing is a relevant moderator of skepticism in the dimensions of concept and application. In addition, professional experience is a relevant determinant as it moderates skepticism's dimensions of concept and academic training. In conclusion, professional experience is a relevant moderator of professional skepticism, such that practical activities in auditing are a strategy that can favor and enhance the training of future graduates.
\end{abstract}




\section{INTRODUCCIÓN}

El escepticismo profesional es una competencia relevante en la formación de contadores auditores, pues resulta en un elemento esencial en las decisiones del profesional y una de las causas más frecuentes de error en auditoría (Favere-Marchesi y Emby, 2017; Díaz et al., 2020). De acuerdo a las Normas Internacionales de Auditoría (NIA, por sus siglas en inglés), el escepticismo profesional es una "actitud que implica una mentalidad inquisitiva, una especial atención a las circunstancias que pueden ser indicativas de posibles incorrecciones debidas a errores o fraudes, y una valoración crítica de la evidencia de auditoría" (IAASB, 2017). Así, el escepticismo puede entenderse como la actitud que incluye elementos como el cuestionamiento y evaluación critica de las pruebas en la auditoría (PCAOB, 2012). De hecho, autores como Dela Cruz et al. (2020) lo han vinculado con habilidades relacionadas con la capacidad de discernir y evaluar la relevancia y confiabilidad de las evidencias y la información. Lo anterior, ha hecho que el estudio del escepticismo en los profesionales de esta área siga siendo un tema relevante de estudiar en la formación universitaria (Hurtt et al., 2013).

En este contexto, resulta relevante su estudio pues autores como Brown et al. (2016) o Verwey y Asare (2020) señalan que no actuar con el debido escepticismo profesional puede llevar a los auditores a situaciones relacionadas con fraudes, fallas éticas u otros de índole legal, además de que su desarrollo suele ser un indicador relevante de la calidad de la formación del auditor. Es más, estudios recientes señalan que esta temática aplicada en la formación de profesionales es un tema emergente para la comprensión y mejoramiento de la educación de los auditores y su desempeño futuro en cuanto a la calidad de las auditorías realizadas (Khan y Harding, 2020). Tradicionalmente, el escepticismo profesional ha sido analizado desde una perspectiva neutral, donde el auditor mantiene una actitud imparcial sobre la evidencia o bien, de presunción de duda (Nelson, 2009). Así, los auditores en ejercicio profesional se pueden enmarcar en evaluaciones en términos de deshonestidad, lo que comúnmente relacionamos con el escepticismo, o bien honestidad, respecto de las evidencias e información (Bowlin et al., 2015).

Desde el punto de vista de la formación, las Normas Internacionales de Formación para el Contador Profesional (IES, por sus siglas en inglés) en apoyo del desarrollo de estándares en educación desarrolladas por parte del Consejo de Normas Internacionales de Formación en Contaduría (IAESB, por sus siglas en inglés) señalan la importancia de desarrollar competencias profesionales y éticas en auditoría mediante el desarrollo y aplicación del escepticismo profesional (IFAC, 2018). Desde esta perspectiva, autores como Shaub (2020) proponen la importancia de estudiar las variables que interfieren en la formación de los auditores dado que resulta clave la formación del razonamiento ético en los futuros profesionales. Así, complementario a la formación tanto técnica como disciplinar del contador auditor, resulta relevante el análisis desde las habilidades y actitudes éticas que favorecen un adecuado comportamiento profesional puesto que suponen ser cruciales en el ejercicio profesional (Viloria-Ortega y Espinosa-Díaz, 2020).

En relación con lo anterior, autores como Knechel et al. (2012) son enfáticos en señalar que el escepticismo es una competencia relevante en la calidad de las auditorías que el profesional realiza, por lo cual es apreciable su análisis desde la formación universitaria. En tanto, trabajos como el realizado por Nelson (2009) indican que el escepticismo profesional aplica a todas las decisiones que un profesional de esta área emplea durante su ejercicio profesional. Así, los estudios son concluyentes en la importancia que tiene el escepticismo profesional sobre el ejercicio de los auditores considerando que esta competencia es omnipresente a toda norma en auditoría (Nolder y Kadous, 2018). Es más, el escepticismo ha sido considerado una variable relevante en el desarrollo profesional y la estabilidad laboral de los auditores (Cohen et al., 2017).

En ese marco, la formación de estos profesionales es transcendental pues las competencias desarrolladas durante su preparación podrían moderar su grado de escepticismo y la rigurosidad con la que se enfrentan a los problemas en auditoría (Rasso, 2015). Igualmente, algunos estudios han considerado que es una habilidad relevante en el ejercicio profesional y el buen desempeño de los graduados (Septian y Astika, 2019). De hecho, el mismo autor es sentencioso en señalar que el desarrollo de esta habilidad y competencia es una señal clave de la formación de un profesional de esta área, dado que le permite discernir claramente de las evidencias bajo el principio de objetividad. Respecto de la formación profesional de los auditores, autores como Rodgers et al. (2017) han analizado el impacto de la transferencia de conocimiento sobre la precisión en los juicios de los auditores concluyendo significancia de esta relación. Por lo anterior, es que el estudio del desarrollo de habilidades como el escepticismo profesional durante la formación profesional es revelador, pues permite implementar mejoras a nivel curricular que permita a los egresados desarrollar un mejor grado de escepticismo en su ejercicio laboral futuro.

A nivel internacional, los estudios coinciden en que el escepticismo profesional y su desarrollo debe ser analizado a nivel individual (Gul et al., 2013). No obstante, otros estudios señalan que existen factores individuales y también, situacionales o contextuales (Septian y Astika, 2019). Sobre este punto, el presente 
estudio propone estudiar los determinantes del grado de escepticismo profesional en los egresados de auditoría a partir de aspectos individuales, pero también a nivel situacional incluyendo variables como: el sexo, la experiencia laboral, el sector público-privado en que se desarrolla, entre otros. A nivel individual, autores como Brown et al. (2016) han analizado la existencia de diferencias significativas por sexo y experiencia laboral en la calidad de las auditorías y sus implicancias prácticas.

Por otro lado, a nivel situacional o contextual, estudios como el desarrollado por Lee et al. (2016) han concluido que cualquier experiencia vinculada con la profesionalización en auditoria tiende a influir positivamente sobre la conciencia profesional. Además, otros autores han considerado relevante que la experiencia laboral puede influir sobre la calidad de las auditorias y el grado de escepticismo que desarrollan los profesionales (Koch et al., 2016). Inclusive, trabajos como el realizado por Brown et al. (2016) son concluyentes en analizar que la experiencia laboral en auditoría produce impactos positivos sobre los componentes de la competencia profesional de escepticismo. Del mismo modo, se ha considerado relevante analizar como el contexto de la empresa puede afectar el escepticismo profesional donde las características económicas o de dependencia público-privado pueden impactar en la percepción del profesional (He et al., 2018). Al mismo tiempo, estudios recientes han considerado relevante la incorporación de variables de medición tales como autoeficacia, inteligencia emocional, socialización anticipatoria, entre otros (Farag y Elias, 2016; Yang et al., 2018). En efecto, los estudios desarrollados lo han vinculado también con el desarrollo de variables de índole cognitivo (Peytcheva, 2014). Estos aspectos no son abordados por el presente estudio, pero resultan en interesantes líneas futuras de desarrollo en la problemática.

Esta investigación tuvo por objetivo determinar los factores que influyen en la percepción de escepticismo en los egresados de Auditoría. En particular, se realizó una caracterización de la muestra y posteriormente, se analizó los determinantes para el grado de conocimiento, aplicación y formación en escepticismo profesional en la muestra de ex alumnos de la Universidad Católica del Maule. La metodología empleada fue la estimación de un modelo probit ordenado a través de una variable latente que mide el grado de percepción del escepticismo profesional de los egresados de auditoría. Lo anterior, resulta en una contribución importante a nivel científico desde la perspectiva de la formación de contadores auditores, considerando que permite evidenciar los determinantes de la percepción sobre escepticismo profesional en los graduados.

\section{METODOLOGÍA}

El presente estudio es de tipo descriptivo y de corte transversal a través de la aplicación de una encuesta a egresados de la carrera de Contador Auditor de la Universidad Católica del Maule. Las siguientes secciones muestran en específico la muestra, el instrumento y metodología utilizada para el procesamiento de los datos.

\section{Participantes y muestra}

Los participantes corresponden a egresados de diferentes cohortes de la carrera de Contador Auditor de la Universidad Católica del Maule. La muestra fue calculada de manera probabilística al 95\% de confianza y 5\% de margen de error máximo admisible. Se consideró una muestra objetivo finita de 256 egresados de la carrera, obteniendo que la muestra debía ser de 154 participantes. Finalmente, se logró aplicar el cuestionario en modalidad de auto-reporte a 160 ex alumnos de la carrera. A continuación, se muestra el detalle del cálculo de la muestra.

$$
\mathrm{n}=\frac{\mathrm{N} \cdot \mathrm{z}_{\mathrm{a}}^{2} \cdot \mathrm{p} \cdot(1-\mathrm{p})}{\mathrm{d}^{2} \cdot(\mathrm{N}-1)+\mathrm{z}_{\mathrm{a}}^{2} \cdot \mathrm{p} \cdot(1-\mathrm{p})}
$$

Donde, $\mathrm{N}$ es el tamaño de la población; $\mathrm{z}_{\mathrm{a}}$ el nivel de confianza; $\mathrm{p}$ la probabilidad de éxito; $q$ la probabilidad de fracaso y d el error máximo admisible.

$$
\mathrm{n}=\frac{256 \cdot 1,96^{2} \cdot 0,5 \cdot(1-0,5)}{0,05^{2} \cdot(256-1)+1,96^{2} \cdot 0,5 \cdot(1-0,5)}=154
$$

La Tabla 1 resume las variables utilizadas en el estudio. Tal como muestra Tabla 1 un $97,5 \%$ de los egresados pertenecen a la malla innovada (por competencias), mientras que un $2,5 \%$ a la malla por objetivos. En el caso de sedes, un 66,6\% corresponden a la Sede 1 (Talca) mientras que un 33,3\% egresaron de la Sede 2 (Curicó). Con respecto al sexo, un $47,5 \%$ de los encuestados son hombres, mientras que un $52,5 \%$ mujeres. El rango de edad predominante es entre 31 y 40 años con un $71,6 \%$ de las respuestas, mientras que un $22,8 \%$ tienen entre 41 y 50 años. De los egresados, un 3,1\% tienen entre 23 a 30 años y el 2,5\% tienen más de 51 años. Referente al área de trabajo, un $9,4 \%$ se dedica al área de Auditoría, un 51,9\% Contabilidad, un 30\% Administración y un 8,8\% en Otra área de desempeño. Los detalles de todas las variables pueden consultarse en la Tabla 1. 
Tabla 1: Caracterización de la muestra

\begin{tabular}{|c|l|c|c|c|l|c|c|}
\hline \multicolumn{2}{|l|}{ Variable } & $N$ & Porcentaje & \multicolumn{2}{|l|}{ Variable } & $N$ & Porcentaje \\
\hline Malla & Innovada (Competencias) & 156 & $97,5 \%$ & Sede & Sede 1 (Talca) & 107 & $66,7 \%$ \\
Curricular & Objetivos & 4 & $2,5 \%$ & & Sede 2 (Curicó) & 53 & $33,3 \%$ \\
\hline Sexo & Hombre & 76 & $47,5 \%$ & Edad & 23 a 30 años & 5 & $3,1 \%$ \\
& Mujer & 84 & $52,5 \%$ & & 31 a 40 años & 115 & $71,6 \%$ \\
& & & & & 41 a 50 años & 36 & $22,8 \%$ \\
& & & & & 51 o más años & 4 & $2,5 \%$ \\
\hline Área de & Auditoría & 15 & $9,4 \%$ & Sector & Público & 8 & $5,0 \%$ \\
Trabajo & Contabilidad & 83 & $51,9 \%$ & de & Privado & 152 & $95,0 \%$ \\
& Administración & 48 & $30,0 \%$ & Trabajo & & & \\
& Otro & 14 & $8,8 \%$ & & & & \\
Experiencia & Menos de 5 años & 18 & $11,1 \%$ & & & & \\
Laboral & Entre 5 y 9 años & 95 & $59,3 \%$ & & & & \\
& Entre 10 y 14 años & 43 & $27,2 \%$ & & & \\
& 15 o más años & 4 & $2,5 \%$ & & & & \\
&
\end{tabular}

Instrumento de medición

El instrumento corresponde a una versión ajustada del desarrollado por Ávila y Maldonado (2015) que tenía por finalidad medir escepticismo relacionado al fraude. Dicho instrumento en su diseño consideró originalmente 96 variables del set de Normas Internacionales de Auditoría. La presente aplicación corresponde a una adaptación de dicho cuestionario con un enfoque en el escepticismo del profesional. El instrumento considera 24 ítems distribuidos en las dimensiones de: 1) conocimiento del concepto de escepticismo profesional; 2) aplicación del escepticismo profesional; y 3) formación académica sobre escepticismo profesional, las que son evaluadas en una escala de Likert de grado de acuerdo (entre 1 y 7 ). Además, en el cuestionario aplicado se consideró un apartado de antecedentes sociodemográficos.

La validez del instrumento fue realizada previa a la aplicación en la muestra mediante la técnica de juicio de expertos, los cuales fueron consultados sobre la suficiencia, claridad, coherencia y relevancia de los ítems en la escala. Posterior a esto, se ajustaron los instrumentos en virtud de los comentarios y sugerencias de los expertos asegurando validez en la medición. Respecto a la confiabilidad y consistencia interna, se obtuvieron indicadores adecuados a nivel general (alpha de cronbach 0,9000) y por dimensiones: 1 ) conocimiento del concepto de escepticismo profesional (alpha de cronbach 0,7020); 2) aplicación del escepticismo profesional (alpha de cronbach 0,8390); y 3) formación académica sobre escepticismo profesional (alpha de cronbach 0,9340).

\section{Modelo probabilístico ordenado}

El método de estimación fue realizado a través de un modelo probabilístico ordenado que asume que la percepción sobre escepticismo profesional de los egresados no se observa de manera directa. Por lo tanto, se considera una variable latente discreta de carácter ordinal a partir de las escalas medidas por el instrumento aplicado. La variable latente esta ordenada de acuerdo a los criterios de: bajo, medio y alto grado de percepción de escepticismo por cada dimensión de evaluación, definidos a partir del porcentaje de acuerdo y utilizando en su construcción los percentiles 50, 75 y el máximo de cada escala como referencia.

El modelo fue estimado a través del método de máxima verosimilitud considerando un modelo no lineal basado en una distribución normal (probit ordenado). Las variables independientes utilizadas fueron: experiencia laboral, malla de egreso, la sede de egreso, sexo del egresado, tramo de edad del egresado, área de trabajo actual y sector en que se desempeña. Siguiendo a Greene (2012) el modelo general está definido por,

$$
y^{*}=x^{\prime} \beta+\varepsilon
$$

En esta ecuación, $y^{*}$ es una variable dependiente que no es directamente observable. Por tanto, lo observado corresponde a,

$$
\begin{aligned}
& y=0 \text { si } y^{*} \leq 0, \\
& y=1 \text { si } 0<y^{*} \leq \mu_{1}, \\
& y=2 \text { si } \mu_{1}<y^{*} \leq \mu_{2}, \\
& y=j \text { si } \mu_{j-1} \leq y^{*}
\end{aligned}
$$


Donde la ecuación 7, j representa a el número de posibles respuestas de la variable latente ordinal. En este caso particular, se tiene que j = 3 probables alternativas (medidas por el grado de percepción de escepticismo: baja, media 0 alta). Las $x$ ' de la ecuación 3 corresponden a las variables independientes definidas anteriormente. En lo que respecta a $\beta$, representa los coeficientes estimados para el modelo probabilístico ordenado, sin embargo, tal como señala Greene (2012) estos coeficientes no pueden ser interpretados en magnitud. Por dicha razón, se muestran los resultados en términos de efectos marginales promedio con errores estándar robustos a la heterocedasticidad mediante el método Delta.

\section{RESULTADOS}

A continuación, se muestran los resultados en términos descriptivos y posteriormente, los resultados del modelo probabilístico ordenado.

\section{Resultados descriptivos}

Los resultados descriptivos se muestran en la Tabla 2. La dimensión conocimiento del concepto de escepticismo en media muestra un valor cercano al $70 \%$ de acuerdo por parte de los egresados con una dispersión de 14,4\% y un valor mediano de 73\%. La dimensión aplicación del escepticismo profesional en media muestra un valor cercano al $74 \%$ de acuerdo por parte de los egresados con una dispersión de 14,4\% y un valor mediano de $75 \%$. La dimensión aplicación del escepticismo profesional en media muestra un valor cercano al $67 \%$ de acuerdo por parte de los egresados con una dispersión de 14,1\% y un valor mediano de $64 \%$. Se puede observar que esta última es la que presenta un menor valor de acuerdo, por lo que realza la importancia de estudiar los efectos de la formación en escepticismo profesional de los egresados.

También, en la Tabla 3 se presentan las correlaciones (los niveles de significancia de correlaciones son: *10\%; ${ }^{* \star} 5 \% ;{ }^{* * *} 1 \%$ ) entre dimensiones mostrando que existen relaciones directas, fuertes y significativas entre estas. Lo anterior, indica una relación positiva entre las diferentes dimensiones que componen el instrumento, es decir, aquellos ex alumnos con altos niveles de percepción respecto de una dimensión específica de escepticismo profesional, también cuentan con un alto grado de percepción en otra dimensión.

Tabla 2: Descriptivos principales por dimensión

\begin{tabular}{|l|c|c|c|c|c|c|}
\hline \multicolumn{1}{|c|}{ Variables } & Media & $\begin{array}{c}\text { Desviación } \\
\text { Estándar }\end{array}$ & Mínimo & Máximo & $\begin{array}{c}\text { Percentil } \\
50\end{array}$ & $\begin{array}{c}\text { Percentil } \\
75\end{array}$ \\
\hline $\begin{array}{l}\text { 1) conocimiento del concepto de } \\
\text { escepticismo profesional }\end{array}$ & 0,7017 & 0,1439 & 0,1400 & 1,000 & 0,7300 & 0,8000 \\
\hline $\begin{array}{l}\text { 2) aplicación del escepticismo } \\
\text { profesional }\end{array}$ & 0,7409 & 0,1426 & 0,1400 & 1,000 & 0,7500 & 0,8400 \\
\hline $\begin{array}{l}\text { 3) formación académica sobre } \\
\text { escepticismo profesional }\end{array}$ & 0,6687 & 0,1411 & 0,1400 & 1,000 & 0,6400 & 0,7600 \\
\hline Escala total & 0,7122 & 0,1256 & 0,1400 & 1,000 & 0,7100 & 0,7800 \\
\hline
\end{tabular}

Tabla 3: Matriz de correlaciones por dimensión

\begin{tabular}{|c|c|c|c|c|}
\hline Variables & $\begin{array}{l}\text { 1) conocimiento del } \\
\text { concepto de } \\
\text { escepticismo } \\
\text { profesional }\end{array}$ & $\begin{array}{l}\text { 2) aplicación del } \\
\text { escepticismo } \\
\text { profesional }\end{array}$ & $\begin{array}{l}\text { 3) formación } \\
\text { académica sobre } \\
\text { escepticismo } \\
\text { profesional }\end{array}$ & Escala total \\
\hline $\begin{array}{l}\text { 1) conocimiento del concepto } \\
\text { de escepticismo profesional }\end{array}$ & 1 & & & \\
\hline $\begin{array}{l}\text { 2) aplicación del escepticismo } \\
\text { profesional }\end{array}$ & $0,8628^{\star * *}$ & 1 & & \\
\hline $\begin{array}{l}\text { 3) formación académica sobre } \\
\text { escepticismo profesional }\end{array}$ & $0,5724^{\star \star *}$ & $0,4994^{* * *}$ & 1 & \\
\hline Escala total & $0,9351^{* * *}$ & $0,9411^{* * *}$ & $0,7294^{* * *}$ & 1 \\
\hline
\end{tabular}

\section{Resultados modelo probabilístico ordenado}

Los resultados del modelo ordenado en efectos marginales se muestran en la Tabla 4 (en paréntesis se muestra errores estándar robustos por método Delta, mientras que los niveles de significancia de las pruebas z son: ${ }^{*} 10 \% ;{ }^{* \star} 5 \% ;{ }^{* * \star} 1 \%$ ). En términos de ajuste, el modelo ordenado para el caso de la variable dependiente: 1) conocimiento del concepto escepticismo profesional muestra un pseudo $R$ cuadrado del orden del $5,98 \%$; 
2) aplicación del escepticismo profesional un 9,64\%; y 3) formación académica sobre escepticismo profesional un $9,05 \%$, lo que resulta adecuado para el tipo de modelo empleado. Los resultados obtenidos se detallan a continuación, denotando que existen diferencias entre los determinantes que influencian el grado de percepción sobre escepticismo profesional en las dimensiones de concepto, aplicación y formación académica.

Al analizar la dimensión de percepción sobre conocimiento del concepto de escepticismo, los resultados muestran que el área de trabajo o desempeño actual afecta significativa y positivamente el pertenecer al grupo de ex alumnos con alto grado de escepticismo. En este sentido, aquellos egresados que trabajan en el área de auditoría poseen una percepción de acuerdo alta con una probabilidad del $55 \%$ con respecto a aquellos que trabajan en otras áreas. El sector donde se desempeñan también afecta significativamente la percepción de conocimiento del concepto de escepticismo, notando que aquellos que trabajan en el sector privado tienen un $28 \%$ menos de probabilidades de tener una alta percepción de acuerdo con el conocimiento del concepto de escepticismo. Conjuntamente, aquellos que poseen una experiencia mayor muestran que la probabilidad de encontrarse altamente de acuerdo con el conocimiento del concepto de escepticismo disminuye en torno un $43 \%$. También, se analizaron las variables sede de egreso, género, edad y experiencia laboral, no obstante, se mostraron como no significativos en la explicación del conocimiento del concepto de escepticismo profesional (ver detalles en la Tabla 4).

Tal como en el caso anterior, al analizar la dimensión de percepción sobre la aplicación del escepticismo en el ejercicio profesional, se muestra que el área de trabajo actual es una variable significativa. En este contexto, se logra apreciar que trabajar en el área de auditoría aumenta la probabilidad de tener un alto grado de acuerdo con probabilidad en torno al $70 \%$, mientras que en el área de contabilidad la probabilidad es de un $40 \%$ aproximadamente. Para el caso de aquellos que trabajan en el rubro de administración las diferencias no se muestran como significativas, lo que alude probablemente a la naturaleza de las funciones que desempeñan en su ejercicio profesional. En cuanto a la percepción media sobre aplicación del escepticismo, trabajar en auditoría o contabilidad reduce la probabilidad en el orden del $55 \%$ y $27 \%$ respectivamente, dado que ambas áreas son concordantes en señalar alto grado de percepción respecto al escepticismo en el ejercicio profesional. Este resultado tiene directa relación con las funciones desempeñadas por los profesionales, puesto que son los que ofician en las áreas de contabilidad y auditoría los que se ven mayormente expuestos a la aplicación del escepticismo.

En contraparte, el sector de trabajo privado muestra que decae la probabilidad en torno al $30 \%$ si solo tomamos como referencia el alto grado de acuerdo con respecto a la aplicación del escepticismo profesional. Sin embargo, al considerar un grado de acuerdo medio la probabilidad de este sector aumenta en un $23 \%$. Es decir, aquellos ex alumnos empleados en el sector privado muestran menor grado de percepción sobre la aplicación del escepticismo profesional. En este caso, además se observan diferencias entre las sedes de egreso. Así, aquellos egresados de la Sede Talca tienen un $24 \%$ menos de probabilidad de percibir un alto grado de percepción de la aplicación del escepticismo profesional en su oficio. Esta probabilidad es significativa en todos los niveles de percepción, siempre favorable de los formados en la Sede Curicó, lo que resulta interesante desde un punto de vista de la implementación del plan de estudios o del futuro ejercicio profesional de los egresados. Al igual que el caso anterior, se analizaron también las variables referidas al género y tramo de edad, sin embargo, no se consignan diferencias significativas sobre el grado de percepción sobre la aplicación del escepticismo profesional.

Respecto del grado de acuerdo con la percepción sobre la formación académica sobre escepticismo profesional, las variables que se muestran significativas son la sede de egreso, con un efecto en torno al $14 \%$ favorable a la Sede Curicó. En los casos de percepción baja y media respecto a la formación en escepticismo, los efectos se muestran en torno al 5\% y $9 \%$ para el caso de los egresados de la Sede Talca. Esta situación, al igual que la de aplicación del concepto de escepticismo es interesante desde el punto de vista de la implementación curricular. De igual forma, el tramo de edad muestra que, para edades superiores el grado de acuerdo con la formación académica sobre escepticismo es mayor. Para el caso de alto grado de acuerdo, el tramo entre 31 y 40 años obtiene un efecto marginal de $21 \%$, mientras que el tramo de sobre 41 años un efecto del $27 \%$ sobre la probabilidad de encontrarse altamente conforme con la formación en escepticismo recibida. Además, para el caso de mediano grado de acuerdo se puede observar que el sector privado muestra un efecto significativo del $4 \%$ de aumento de probabilidad con respecto a la formación Finalmente, el bajo nivel de acuerdo se asocia a egresados con 9 o menos años de experiencia laboral con un efecto en torno al $6 \%$, lo que releva nuevamente la importancia de la experiencia como moderador del grado de percepción sobre escepticismo profesional en los contadores auditores. En el caso de la variable género, no se reportan efectos marginales significativos con respecto al grado de percepción sobre la formación en escepticismo profesional. 
Tabla 4: Resultados efectos marginales promedio modelo ordenado

\begin{tabular}{|c|c|c|c|c|c|c|c|c|c|}
\hline \multirow[t]{2}{*}{ Variables } & \multicolumn{3}{|c|}{$\begin{array}{l}\text { Conocimiento del concepto } \\
\text { escepticismo profesional }\end{array}$} & \multicolumn{3}{|c|}{$\begin{array}{c}\text { Aplicación del escepticismo } \\
\text { profesional }\end{array}$} & \multicolumn{3}{|c|}{$\begin{array}{c}\text { Formación académica sobre } \\
\text { escepticismo profesional }\end{array}$} \\
\hline & Bajo & Medio & Alto & Bajo & Medio & Alto & Bajo & Medio & Alto \\
\hline \multirow{3}{*}{ Sede (Talca $=1)$} & \multirow{2}{*}{0,0056} & \multirow{2}{*}{0,0268} & \multirow{2}{*}{$-0,0324$} & 0,0227 & 0,2169 & $-0,2394$ & 0,0512 & 0,0895 & $-0,1407$ \\
\hline & & & & 7 & & 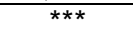 & & & \\
\hline & $(0$ & $(0,0693)$ & $(0,0841)$ & $(0,0135)$ & $(0,0678)$ & $(0,0758)$ & $0,0276)$ & $(0,0440)$ & $0,0679)$ \\
\hline \multirow{2}{*}{ Género (Hombre=1) } & & & & $-0,0087$ & $-0,0690$ & 0,0779 & $-0,0343$ & $-0,0477$ & 0,0820 \\
\hline & $(0,0144)$ & $(0,0588)$ & $(0,0727)$ & $(0,0105)$ & $(0,0676)$ & $(0,0773)$ & $(0,0270)$ & $(0,0354)$ & $(0,0605)$ \\
\hline \multicolumn{10}{|c|}{ Edad (base 51 o más años) } \\
\hline \multirow{2}{*}{ Entre 23 y 30 años } & 0,0167 & 0,0872 & $-0,1038$ & $-0,0046$ & $-0,050$ & 0,0548 & $-0,2043$ & 0,1369 & 0,0674 \\
\hline & $(0,0501)$ & $(0,258)$ & $(0,3052)$ & $(0,0244)$ & $(0,2635)$ & $(0,2875)$ & $(0,2644)$ & $(0,2123)$ & $(0,0671)$ \\
\hline \multirow{2}{*}{ Entre 31 y 40 años } & 0,0126 & 0,0711 & $-0,0837$ & 0,0010 & 0,009 & $-0,0098$ & $-0,3253$ & 0,1124 & $\frac{0,2129}{* \star *}$ \\
\hline & $(0,0299)$ & $(0,21$ & $(0,2448)$ & $(0,0201)$ & 01 & $(0,2092)$ & $(0,2532)$ & $(0,2086)$ & $(0,0582)$ \\
\hline \multirow{2}{*}{ Entre 41 y 50 años } & 0,0045 & 0,0300 & $-0,0345$ & 0,0120 & 0,085 & $-0,0969$ & $-0,3448$ & 0,0755 & 0,2692 \\
\hline & $(0,0299)$ & $(0,2184)$ & $(0,2482)$ & $(0,0232)$ & $(0,1945)$ & $(0,2167)$ & $(0,2505)$ & $\begin{array}{l}(0,2169) \\
\end{array}$ & $(0,0731)$ \\
\hline \multicolumn{10}{|l|}{ Área } \\
\hline \multirow{2}{*}{ Auditoría } & \multirow[t]{2}{*}{$-0,0743$} & $\frac{-0,4707}{* \star}$ & $\frac{0,5449}{*}$ & $-0,1475$ & \begin{tabular}{|l|}
$-0,5479$ \\
\end{tabular} & 0,6954 & \multirow{2}{*}{$-0,0102$} & $-0,0269$ & 0,0370 \\
\hline & & $(0,2192)$ & $(0,3276)$ & 1899) & $(0,0636)$ & $(0,201)$ & & $(0,3546)$ & $0,4905)$ \\
\hline \multirow{2}{*}{ Contabilidad } & $-0,0507$ & $-0,1323$ & 0,1830 & $-0,1335$ & $-0,2680$ & 0,4016 & 0,0113 & 0,0216 & $-0,0329$ \\
\hline & $(0,1112)$ & \begin{tabular}{|l}
$(0,1243)$ \\
\end{tabular} & $(0,2337)$ & $(0,1824)$ & \begin{tabular}{|l|}
$(0,0477)$ \\
\end{tabular} & $(0,1794)$ & $(0,1009)$ & $(0,2144)$ & $(0,3152)$ \\
\hline & & 0,0078 & $-0,0$ & $-0,0337$ & $\mid-0,014$ & 0,0472 & 0,0514 & 0,0601 & $\frac{-0,1114}{-114}$ \\
\hline & $(0,0628)$ & $(0,0761)$ & $(0,1383)$ & $(0,1152)$ & $(0,0271)$ & $(0,1254)$ & $(0,0555)$ & $(0,1619)$ & $(0,2029)$ \\
\hline Sectc & 0,1341 & 0,1458 & $\frac{-0,2799}{* \star \star}$ & 0,0740 & 0,2285 & $-0,3025$ & 0,0540 & $\frac{0,0384}{*}$ & $-0,0924$ \\
\hline & $(0,1139)$ & $(0,0461)$ & $(0,0894)$ & 0474) & $(0,0602)$ & $(0,0926)$ & $(0,0848)$ & $(0,0236)$ & $(0,1040)$ \\
\hline houglth & menos d & e 5 años) & & & & & & & \\
\hline & 0,0041 & 0,0239 & $-0,0280$ & 0,0117 & 0,1167 & $-0,1284$ & $\underset{* \star *}{0,0629}$ & 0,2462 & $-0,3091$ \\
\hline & & $(0,1$ & $(0,21$ & $(0,0$ & $(0,1491)$ & $(0,1$ & $(0,0239)$ & $\overline{(0,26}$ & $(0,2811)$ \\
\hline & & 0 & $-0,1$ & 0,0595 & 0,2981 & $-0,3576$ & 0,0782 & 0,2605 & $-0,3387$ \\
\hline & $(0,0858)$ & $(0,2498)$ & $(0,3341)$ & $(0,0818)$ & $(0,2195)$ & $(0,2978)$ & $(0,0872)$ & $(0,322)$ & $(0,4049)$ \\
\hline & 0,4764 & $-0,0$ & $-0,4274$ & $-0,0085$ & $-0,278$ & 0,2866 & 0,0450 & 0,2185 & 0,2635 \\
\hline & $(0,4419)$ & $(0,2245)$ & $(0,265)$ & $(0,0066)$ & \begin{tabular}{|l|}
$(0,1796)$ \\
\end{tabular} & $(0,1842)$ & $(0,1703$ & $(0,5638)$ & $(0,7325)$ \\
\hline & & & & & & & & & \\
\hline & & $-1,17 ?$ & & & $-1,111,46$ & & & $-1,16$ & \\
\hline & & 160 & & & 160 & & & 160 & \\
\hline Pseudo R² & & 0,0598 & & & 0,0964 & & & 0,0905 & \\
\hline
\end{tabular}

\section{DISCUSIÓN}

Este estudio aporta evidencia empírica sobre la formación de auditores con respecto al desarrollo de competencias relacionadas con el escepticismo profesional. Desde el punto de vista formativo, estas competencias son relevantes en la educación de los auditores, considerando los estándares en educación desarrolladas por parte del Consejo de Normas Internacionales de Formación en Contaduría (IAESB) y su relación con los componentes éticos involucrados en el ejercicio profesional de los auditores. Los resultados sugieren una serie de implicancias prácticas en la formación profesional de los futuros auditores, considerando que autores como Dela Cruz et al. (2020) o Rodgers et al. (2017) relevan la importancia de la formación sobre las competencias relacionadas al escepticismo profesional. Las implicancias prácticas y su discusión, se presentan a continuación.

En primer lugar, se puede notar la existencia de diferencias entre las sedes. Esto se produce principalmente en las dimensiones de aplicación y formación sobre escepticismo lo que puede obedecer a: 1) la formación recibida por los egresados (malla innovada, prácticas disciplinares implementadas, prácticas profesionales desarrolladas, etc.); o bien, 2) a que los egresados de las sedes se desempeñan en distintas áreas posterior al egreso (algunos más centrados en auditoría y otros en áreas como contabilidad o administración). Estos resultados son interesantes de analizar desde el punto de vista de la implementación curricular y necesariamente sugieren líneas futuras de investigación que vinculen la implementación práctica del plan de estudios con el desarrollo de competencias profesionales referidas al escepticismo. 
En segundo lugar, se nota que la edad muestra efectos positivos respecto de la formación académica recibida, es decir los egresados con mayor edad tienden a percibir de mejor manera la formación sobre escepticismo recibida. Intuitivamente se denota que el escepticismo profesional se desarrolla con el ejercicio profesional y la práctica, por lo cual el resultado parece estar vinculado a la experiencia, además que autores como Brown et al. (2016) señalan la relevancia de la experiencia y la edad sobre el desarrollo del escepticismo en los profesionales de auditoría. También, este efecto de transferencia del conocimiento por experiencia ha sido considerado relevante en estudios internacionales como es el caso de Rodgers et al. (2017). Lo anterior, es apreciable pues la evidencia empírica muestra que la exposición de los auditores a estrictos análisis favorece la generación de habilidades de escepticismo profesional (Rasso, 2015).

En tercer lugar, se releva que el área de desempeño es la variable más relevante y se muestra significativa en la explicación del escepticismo. En otras palabras, trabajar en auditoría aumenta la percepción y grado de acuerdo con respecto a la aplicación del escepticismo profesional. Este caso igual sucede con los egresados que se desempeñan en el área de contabilidad, pero en menor magnitud, lo que sugiere que el ámbito de desempeño modifica la percepción sobre el escepticismo. Con respecto al conocimiento del concepto de escepticismo también se observan diferencias importantes de aquellos egresados que se desempeñan en el área de auditoría. Estos resultados son vinculantes con la evidencia en la literatura internacional que ha estudiado el impacto del aprendizaje por el ejercicio profesional y el ámbito de desempeño sobre el escepticismo (Lee et al., 2016; Koch et al., 2016).

Los resultados de este estudio permiten evidenciar que el ejercicio profesional modifica las percepciones de los egresados respecto del escepticismo, tal como sugieren Septian y Astika (2019). También, autores como Lee et al. (2016) proponen que las experiencias prácticas en auditoria modifican favorablemente el desarrollo de la competencia en escepticismo, lo que es relevado en este estudio. Lo anterior, es un indicio significativo para el desarrollo de estrategias prácticas en la formación de los estudiantes en temas aplicados al escepticismo profesional, tales como prácticas en auditoría, vivencias o estudios de casos aplicados, que favorezcan la retroalimentación y el aprendizaje durante el proceso formativo.

Para el diseño evaluativo y del aprendizaje de esta competencia, se considera relevante hacer uso de estrategias desde la planeación, recolección y análisis de información, además de la integración de la aplicación de prácticas en auditoría. Esto tomando en consideración que autores como Martelo et al. (2018) indican que a través de estas estrategias se enriquece el aprendizaje práctico y se favorece el mejoramiento de procesos puntuales en los distintos perfiles de estudiantes. Por lo anterior, es que las experiencias y desempeño en auditoría resultan en un moderador del desarrollo de la habilidades y competencias tanto conceptuales como de aplicación sobre escepticismo profesional, las que deberían ser potenciadas en los planes formativos de los estudiantes. Es por ello, que tanto las experiencias prácticas, así como aplicaciones en auditoría deberían ser potenciadas tempranamente en la formación profesional de los contadores auditores.

\section{CONCLUSIONES}

De acuerdo a los resultados del estudio y la discusión realizada, se pueden resumir los siguientes hallazgos: 1) respecto de la dimensión de conocimiento del concepto escepticismo profesional, su desarrollo se ve moderado por el área de desempeño en auditoría y la experiencia profesional; 2) respecto de la dimensión de aplicación del concepto escepticismo profesional, su desarrollo se ve influenciado por el área de desempeño profesional en auditoría; y 3) respecto de la dimensión de formación académica sobre escepticismo profesional, su desarrollo se ve moderado por la experiencia y la edad del egresado. Lo anterior, permite concluir la importancia de las experiencias prácticas en la formación de auditores, tomando como referencia el vínculo de este último con el ejercicio profesional y el desarrollo de las competencias referidas al escepticismo profesional.

\section{REFERENCIAS}

Ávila, J. A. S., y Maldonado, N. M. R., Modelo Propuesto Para La Detección De Fraudes Por Parte De Los Auditores Internos Basado En Las Normas Internacionales De Auditoría, https://doi.org/10.11144/Javeriana.cc16-42.mpdf, Cuadernos de Contabilidad, 16(42), 579-623, (2015).

Bowlin, K. O., Hobson, J. L., y Piercey, M. D., The Effects of Auditor Rotation, Professional Skepticism, and Interactions with Managers on Audit Quality, https://doi.org/10.2308/accr-51032, The Accounting Review, 90(4), 1363-1393, (2015).

Brown, V. L., Gissel, J. L., y Gordon Neely, D., Audit Quality Indicators: Perceptions of Junior-Level Auditors, https://doi.org/10.1108/MAJ-01-2016-1300, Managerial Auditing Journal, 31(8/9), 949-980, (2016). 
Cohen, J. R., Dalton, D. W., y Harp, N. L. Neutral and Presumptive Doubt Perspectives of Professional Skepticism and Auditor Job Outcomes, https://doi.org/10.1016/j.aos.2017.08.003, Accounting, Organizations and Society, 62, 1-20, (2017).

Dela Cruz, A. L., Patel, C., Ying, S., y Pan, P. The Relevance of Professional Skepticism to Finance Professionals' Socially Responsible Investing Decisions, https://doi.org/10.1016/j.jbef.2020.100299, Journal of Behavioral and Experimental Finance, 26 (2020).

Díaz, Y. S. E., Mantilla, J. T. R., y Sarabia, V. M. B., Nociones del Escepticismo Profesional del Contador Público en Ejercicio de las Auditorías: Una Perspectiva Teórica, https://doi.org/10.22267/rtend.202102.150, Tendencias, 21(2), 266282, (2020).

Farag, M. S., y Elias, R. Z., The Relationship Between Accounting Students' Personality, Professional Skepticism and Anticipatory Socialization, https://doi.org/10.1080/09639284.2015.1118639, Accounting Education, 25(2), 124-138 (2016).

Favere-Marchesi, M., y Emby, C., The Alumni Effect and Professional Skepticism: An Experimental Investigation, https://doi.org/10.2308/acch-51920, Accounting Horizons, 32(1), 53-63, (2017).

Greene, W. H., Econometric Analysis (7th ed.), Prentice Hall, (2012).

Gul, F. A., Wu, D., y Yang, Z., Do Individual Auditors Affect Audit Quality? Evidence from Archival Data, https://doi.org/10.2308/accr-50536, The Accounting Review, 88(6), 1993-2023. (2013).

He, X., Kothari, S. P., Xiao, T., y Zuo, L., Long-Term Impact of Economic Conditions on Auditors' Judgment. https://doi.org/10.2308/accr-52009, The Accounting Review, 93(6), 203-229, (2018).

Hurtt, R. K., Brown-Liburd, H., Earley, C. E., y Krishnamoorthy, G., Research on Auditor Professional Skepticism: Literature Synthesis and Opportunities for Future Research, https://doi.org/10.2308/ajpt-50361, AUDITING: A Journal of Practice \& Theory, 32(1), 45-97, (2013).

International Auditing and Assurance Standards Boards (IAASB). Handbook of International Quality Control, Auditing, Review, Other Assurance and Related Services Pronouncements, 2016-2017 Ed., ISBN: 978-1-60815-318-3, 1, New York, USA, (2017).

International Federation of Accountants (IFAC). Handbook of International Education Pronouncements, International Accounting Education Standards Board, 2017 Ed., ISBN: 978-1-60815-316-9, New York, USA, (2018).

Khan, J., y Harding, N., Facilitating the Application of Auditors' Underlying Skeptical Disposition: The Relationship Between Trait Skepticism and Basic Human Values, https://doi.org/10.1108/ARJ-06-2017-0107, Accounting Research Journal, 33(1), 34-56, (2020).

Knechel, W. R., Krishnan, G. y otros tres autores, Audit Quality: Insights from the Academic Literature, https://doi.org/10.2308/ajpt-50350, AUDITING: A Journal of Practice \& Theory, 32(1), 385-421, (2012).

Koch, C., Köhler, A., y Yankova, K., Professional Skepticism And Auditor Judgment: Does Trait Skepticism Mitigate the Recency Bias?, https://doi.org/10.2139/ssrn.2880653, Social Science Research Network, (2016).

Lee, S.-C., Su, J.-M., y otros tres autores, A Comprehensive Survey of Government Auditors' Self-Efficacy and Professional Development for Improving Audit Quality, https://doi.org/10.1186/s40064-016-2903-0, SpringerPlus, 5(1), 1263, (2016).

Martelo, R., I. Jiménez-Pitre y A. Quintana, Determinación del Perfil Profesional de Estudiantes de Pregrado Aplicando la Técnica de Análisis Comparativo, https://doi.org/10.4067/S0718-07642018000200029 Información Tecnológica, 29(2), 29-40 (2018).

Nelson, M. W., A Model and Literature Review of Professional Skepticism in Auditing,

https://doi.org/10.2308/aud.2009.28.2.1, AUDITING: A Journal of Practice \& Theory, 28(2), 1-34, (2009).

Nolder, C. J., y Kadous, K., Grounding the Professional Skepticism Construct in Mindset and Attitude Theory: A Way Forward, https://doi.org/10.1016/j.aos.2018.03.010, Accounting, Organizations and Society, 67, 1-14, (2018).

Peytcheva, M., Professional Skepticism and Auditor Cognitive Performance in A Hypothesis-Testing Task, https://doi.org/10.1108/MAJ-04-2013-0852, Managerial Auditing Journal, 29(1), 27-49, (2014).

Public Company Accounting Oversight Board (PCAOB). Maintaining and Applying Professional Skepticism in Audits Staff Audit Practice Alert No. 10, Washington, USA, (2012).

Rasso, J. T., Construal Instructions and Professional Skepticism in Evaluating Complex Estimates, https://doi.org/10.1016/j.aos.2015.03.003, Accounting, Organizations and Society, 46, 44-55, (2015).

Rodgers, W., Mubako, G. N., y Hall, L., Knowledge Management: The Effect of Knowledge Transfer on Professional Skepticism in Audit Engagement Planning, https://doi.org/10.1016/j.chb.2016.12.069, Computers in Human Behavior, 70, 564-574, (2017).

Septian, I., y Astika, I. B. P., Halo Effect Moderating: Impact of Professional Skepticism on Auditor Performance. https://doi.org/10.21744/irjmis.v6n4.680, International Research Journal of Management, IT and Social Sciences, 6(4), 189-196, (2019). 
Shaub, M. K., Understanding Professional Skepticism Through an Ethics Lens: A Research Note. Research on Professional Responsibility and Ethics in Accounting by C. Richard Baker (Ed.), 23, 1-21, Emerald Publishing Limited, Adelphi University, USA, (2020).

Viloria-Ortega, N., y Espinosa-Díaz, Y. S., El Escepticismo Profesional del Auditor. Una Visión desde dos Docentes de Contaduría Pública Colombianos, https://doi.org/10.11144/Javeriana.cc21.epav, Cuadernos de Contabilidad, 21, (2020).

Verwey, I. G. F., y Asare, S. K., The Joint Effect of Ethical Idealism and Trait Skepticism on Auditors' Fraud Detection, https://doi.org/10.1007/s10551-020-04718-8, Journal of Business Ethics, (2021).

Yang, L., Brink, A. G., y Wier, B., The Impact of Emotional Intelligence on Auditor Judgment, https://doi.org/10.1111/ijau.12106, International Journal of Auditing, 22(1), 83-97, (2018). 